\title{
Electron 3D crystallography of protein crystals for visualization of charges
}

\author{
Koji Yonekura $^{1}$ \\ ${ }^{1}$ RIKEN SPring-8 Center, Sayo, Japan \\ E-mail: yone@spring8.or.jp
}

Electron crystallography has the potential to analyze crystals of membrane proteins and macromolecular complexes too small or too thin for X-ray crystallography, as electrons are scattered 4 - 5 orders of magnitude more strongly than X-rays. Electron crystallography yields Coulomb potential maps, rather than electron density maps as $\mathrm{X}$-rays do, providing information on charged states of amino-acids and metals. We present such Coulomb potential maps at $3.4 \AA$ and $3.2 \AA$ resolution, respectively, of Ca2+-ATPase and catalase obtained from crystals of just a few layers thick. These maps demonstrate that it is indeed possible to build atomic models from such crystals and charge information is included, often critical in understanding protein function [1].

We also report a suitable treatment of electron scattering factors of charged atoms for refinement of atomic models against cryo-EM maps [2]. The ScatCurve package developed supports various models for parameterization of scattering curves, and the parameter tables can be implemented in major refinement programs in structural biology. We changed partial charge values of charged amino acid in crystal structures in steps of 0.1 for refinement of the atomic models against electron diffraction data from three-dimensional crystals. By exploring a range of partial charges, we found the proper electrostatic setting to produce atomic models with improved statistics and to better reflect experimental data. Structure refinement for single particle analysis also benefits from the more accurate analysis, and our programs could find wide use for model refinement against cryo-EM maps.

[1] Yonekura, K., Kato, K., Ogasawara, M., Tomita, M. \& Toyoshima, C. (2015). Proc. Natl. Acad. Sci. USA, 112, $3368-3373$.

[2] Yonekura, K. \& Maki-Yonekura, S. (2016). J. Appl. Cryst., 49, 1517-1523.

Keywords: Electron 3D crystallography, protein structure, charges 